\title{
Regressed lymphatic vessels develop during corneal repair
}

\author{
Philip M Kelley, Maria M Steele and Richard M Tempero
}

The fate of newly synthesized lymphatic vessels induced by inflammation is poorly understood. To address this question, we designed experiments to determine the morphologic, phenotypic, and functional differences in regressing lymphatic vessels in the context of corneal recovery after an inflammatory response. A suture removal modification was used to induce corneal recovery after suture induced inflammation. We identified an increase in markers of corneal inflammation in sutured cornea that resolved in 14 days after suture removal. Sprouting newly synthesized lymphatic vessels trafficking MHC-II-positive leukocytes were visualized in sutured cornea. Following suture removal and recovery, the visualized lymphatic vessels were thin and fragmented, had bulbous termini, discontinuous expression of CD31 and VE-cadherin, and excluded MHC-II-positive leukocytes. VEGF-A, VEGF-C, and TGF- $\beta$ mRNA levels were increased during corneal recovery, suggesting a complex interaction between lymphangiogenic factors and the mechanisms that regulate corneal recovery. The balance of lymphatic vessel growth and regression is likely to have a central role in the pathogenesis of corneal inflammatory diseases.

Laboratory Investigation (2011) 91, 1643-1651; doi:10.1038/labinvest.2011.121; published online 22 August 2011

KEYWORDS: cornea; lymphangiogenesis; lymphatic

Corneal inflammatory conditions such as abrasion, keratitis, or transplantation are associated with angiogenesis and lymphangiogenesis. There is evidence linking inflammatory conditions and pathologic lymphangiogenesis in corneal ${ }^{1,2}$ and non-corneal ${ }^{3-5}$ model systems. Stimulated lymphatic vessels hypertrophy, sprout, and migrate to accommodate the increased fluid load and leukocyte trafficking in inflamed tissue, potentially contributing to the pathophysiology. The fate of these newly synthesized lymphatic vessels and how they contribute to wound repair mechanisms is poorly understood. This is relevant as regressed lymphatic vessels may have distinct properties that contribute to the afferent arm of host immunity and other locoregional mechanisms of tissue repair and homeostasis. There is evidence that newly synthesized corneal or tracheal lymphatic vessels do not regress and persist after the resolution of an inflammatory process. $^{2,5,6}$ In contrast, there are data to support that lymphatic vessel regression develops in the cornea after inflammation and that these vessels increase the risk of corneal transplant rejection. ${ }^{7,8}$ However, the details of this process are largely unknown.

The goals of this study were to determine whether lymphatic vessels regress during corneal wound healing, and if so, characterize the morphology and functional properties of regressed lymphatic vessels. The corneal suture model was used to induce corneal inflammation and lymphangiogenesis. We developed a suture removal modification that stimulated corneal repair and recovery. This strategy allowed us to induce lymphangiogenesis by suture placement and study the fate of newly synthesized lymphatic vessels during corneal recovery following suture removal. Here, we provide evidence demonstrating an orderly progressive program of lymphatic vessels regression during corneal recovery. Lymphatic vessels undergoing regression have a characteristic morphology, unique cell surface receptor expression, and do not transport MHC-II-positive leukocytes. Thus, these vessels appear to be functionally unique from newly synthesized lymphatic vessels.

\section{MATERIALS AND METHODS Corneal Suture and Suture Removal Mouse Model}

All animal protocols were approved by Boys Town National Research Hospital Institutional Animal Care and Use Committee in accordance with the NIH guidelines. 129/SV mice were purchased from Jackson Labs (Bar Harbor, MA, USA). Groups of 5-7 gender-matched 129/SV mice aged 
6-10 weeks were used in the experimental studies. We anesthetized mice with ketamine $(100 \mathrm{mg} / \mathrm{kg})$, xylazine $(10 \mathrm{mg} / \mathrm{kg})$, and topical proparacaine $(0.5 \%$ solution $)$. We placed four 10-0 nylon sutures in each quadrant of the right cornea: the outer point of the suture was equidistant from the limbus and the central cornea. Immediately after surgery, we applied gentamicin ointment $(0.3 \%)$ to the eye. We harvested the eyes 7 days after suture placement. In other groups of mice, we removed the sutures after 7 days and harvested the eyes 7,14 , or 28 days after suture removal. Eyes were fixed with 1\% PFA in PBS pH 7.4 then hemisected for whole mount staining or frozen intact in optimal cutting temperature media (Tissue Tek) at $-80{ }^{\circ} \mathrm{C}$.

\section{Corneal H\&E Staining and Analysis}

In all, $10 \mu \mathrm{m}$ sections were cut from frozen eyes and mounted on glass slides. Tissue was stained using standard hematoxylin and eosin (H\&E) techniques. We used light microscopy to visualize the cornea. Images were obtained. In the center of the cornea, we measured corneal thickness and in two fields $(\times 400)$ adjacent to the center cornea we quantified leukocytes.

\section{Inflammatory Cytokine Array}

We made corneal protein extracts in $100 \mu \mathrm{l} 1 \times$ lysis buffer (Raybiotech ELH-Lysis) with mammalian protease inhibitor (Sigma, P8430). A multiplexed sandwich ELISA-based cytokine protein array was used to quantify 20 different proinflammatory cytokines according to the manufacture's recommendations (RayBiotech, Norcross, GA, USA). The glass slide based microarray (Quantibody Mouse Cytokine Array 1, QAM-CYT-1) allowed the analysis of the following cytokines: GM-CSF, IFN $\gamma$, IL- $1 \alpha$, IL-1 $\beta$, IL-2, IL-3, IL-4, IL-5, IL-6, IL-9，IL-10，IL-12，IL-13，IL-17，KC MCP-1，M-CSF, RANTES, TNF- $\alpha$, and VEGF.

\section{Visualization of Lymphatic Vessel Growth and Regression}

Following eye fixation, we excised the corneas and permeablized them with PBS pH 7.4 containing 0.3\% Triton X-100, $0.2 \%$ FCS, and $0.02 \%$ sodium azide (PBS + ) with $5 \%$ FCS for $1 \mathrm{~h}$. We performed antibody incubations with PBS + containing 5\% FCS. We performed all washes with PBS +. We used rat anti-LYVE-1 (Santa Cruz Biotechnology sc-65647; 1:100) or rabbit anti-LYVE-1 (Angio Bio 11-034; $1: 300)$ to stain the lymphatic vasculature, and rat IgM or rabbit IgG (Invitrogen) was used as a negative control. Appropriate secondary antibodies directly conjugated to Alexa555, Alexa488, Alexa633 (Invitrogen), or DyLight488 (Jackson ImmunoResearch Laboratories) were used to detect the primary antibodies. Other primary antibodies used include rat anti-CD31 (BD Biosciences, 550274), hamster anti-CD31 (Millipore, MAB1398Z), rat anti-VEcadherin (eBioscience, 13-1331-81), and rat anti-MHC-II (eBioscience, 14-5321-81). After mounting the corneas on glass slides, we examined the tissue using a Zeiss Axio Imager A.1 microscope. Images were acquired using SPOT Image Analysis. Confocal images were acquired on a Zeiss LSM510 META microscope using a Plan Neo $\times 40$ oil 1.3 n.a. differential interference contrast (DIC) or a Plan $\times 631.4$ n.a. DIC lens.

\section{Morphometric Analysis of Corneal Lymphatic Vessel Growth and Regression}

We recorded immunofluorescence (IF) images from unsutured, sutured, and 14- or 28-day recovered cornea. For each corneal image, we overlaid a rectangular grid (customized by our laboratory) measuring $200 \times 400 \mu \mathrm{m}^{2}$, on the image adjacent to the constitutive limbal lymphatic vessels. Each square within the grid was $40 \mu \mathrm{m}$ on a side. For lymphatic density measurements, we counted the number of grid squares occupied by a lymphatic vessel wall, lumen, or sprout, and we expressed the data as percentage of occupied grid squares (relative area) \pm s.d. To quantify the number of lymphatic vessel sprouts, we counted the number of sprouts within the grid. We expressed the data as a mean number of sprouts/grid \pm s.d. We defined a sprout as a terminal conical projection with a length of least $10 \mu \mathrm{m}$. Regions of decreased LYVE-1 immune-staining were counted in random $\mathrm{mm}$ segments of newly synthesized or regressed corneal lymphatics using the measurement function of Image Pro Plus software (MediaCybernetics, Bethesda, MD, USA). Buttons were defined as interrupted segments of CD31 or VE-cadherin labeling $(<3.2 \mu \mathrm{m})$ outlining lymphatic vessels. ${ }^{9}$ Projected images of newly synthesized or regressed lymphatic vessels stained with LYVE-1 and CD31 or LYVE-1 and VEcadherin were obtained using the Zeiss LSM510 confocal microscope $(\times 400)$. Lymphatic vessel area was calculated. The number of CD31 or VE-cadherin buttons was quantified. The data were expressed as mean number of CD31 or VE-cadherin buttons \pm s.d./ $1000 \mu \mathrm{m}^{2}$ of projected lymphatic vessel.

\section{Quantitative Analysis of MHC-II-Positive Cells in the Cornea}

In all, $10 \mu \mathrm{m}$ frozen sections of mouse cornea were immunostained for MHC-II. MHC-II-positive cells in two $\times 400$ fields adjacent to a suture or in a comparable region per individual were counted. In studies designed to identify the anatomic relationship between lymphatic vessels and MHC-II cells, whole mount corneas were immunostained for LYVE-1 and MHC-II. Stacked confocal microscopy images corresponding to $\sim 20 \times 318 \times 318 \mu \mathrm{m}^{3}$ were obtained. We counted the number of MHC-II-positive cells inside or in contact with LYVE-1-positive vessels in individual lymphatic vessels from a single cornea to demonstrate vessel-to-vessel variability (Figure $7 \mathrm{~b}$ ) or in two corneal fields obtained from sutured, or 14-day recovery groups of four mice (Figure 7c). 


\section{Quantitative RT-PCR}

RNA was isolated from whole mouse unsutured, sutured, or 14-day recovered corneas using TRIzol Reagent as per the manufacturer's instructions (Invitrogen). In all, $300 \mathrm{ng}$ of corneal RNA was reverse transcribed using Superscript III First Strand Synthesis System (Invitrogen). cDNA was amplified in triplicate reactions using TaqMan Gene Expression Master Mix and TaqMan-based probes and primers (Applied Biosystems). Data were normalized to glyceraldehyde 3-phosphate dehydrogenase (GAPDH). Mean fold induction of mRNA levels relative to unsutured cornea was shown. The error bars demonstrate s.d.

\section{Statistical Analysis}

In all histograms the error bars show s.d. A one-way analysis of variance was used followed by Dunnett's multiple comparison test to compare the control group (denoted with ${ }^{\star}$ ) with experimental groups. Brackets between control and experimental groups show a $P$-value $<0.05$.

\section{RESULTS}

\section{Suture and Suture Removal Model of Corneal Inflammation and Repair}

We evaluated normal cornea (referred as no suture or unsutured), sutured, or suture removed cornea by $\mathrm{H} \& \mathrm{E}$ staining. A significant increase in corneal thickness and leukocyte infiltration was identified in sutured cornea that decreased after suture removal (Figures 1a-e). Higher power microscopy revealed hypertrophy of corneal stroma and leukocyte infiltrate in sutured cornea (Figure 1b1). A cytokine protein array using extracts of total cornea demonstrated that corneal suture placement induced expression of several pro-inflammatory or angiogenic cytokines (IL- $1 \alpha$, IL-6, KC, MCP-1, and VEGF). These cytokines were undetectable or present at lower levels in unsutured cornea or suture removed cornea followed by a 14-day recovery (Figures $2 \mathrm{a}-\mathrm{e}$ ). Although other cytokines were evaluated in the array, we did not detect GM-CSF, IFN $\gamma$, IL- $1 \beta$, IL-2, IL-3, IL-4, IL-5, IL-9, IL-10, IL-12, IL-13, IL-17, M-CSF, RANTES, or TNF- $\alpha$ in cornea extracts from unsutured, sutured, or 14day recovery mice. These data established that suture placement for 7 days induced corneal inflammation and identified a recovery phase (14 days after suture removal) with resolution of corneal inflammation. These findings allowed us to explore the fate of newly synthesized lymphatic vessels in cornea recovered from an episode of inflammation.

\section{Visualization of Corneal Lymphatic and Blood Vessels}

We labeled unsutured, sutured, 14-, or 28-day recovered cornea with antibodies to LYVE-1 to detect lymphatic vessels or CD31 (PECAM) a panendothelial marker. Limbal blood and lymphatic vessels were visualized with IF microscopy (Figures $3 \mathrm{a}$ and e). Corneal sutures induced lymphatic and
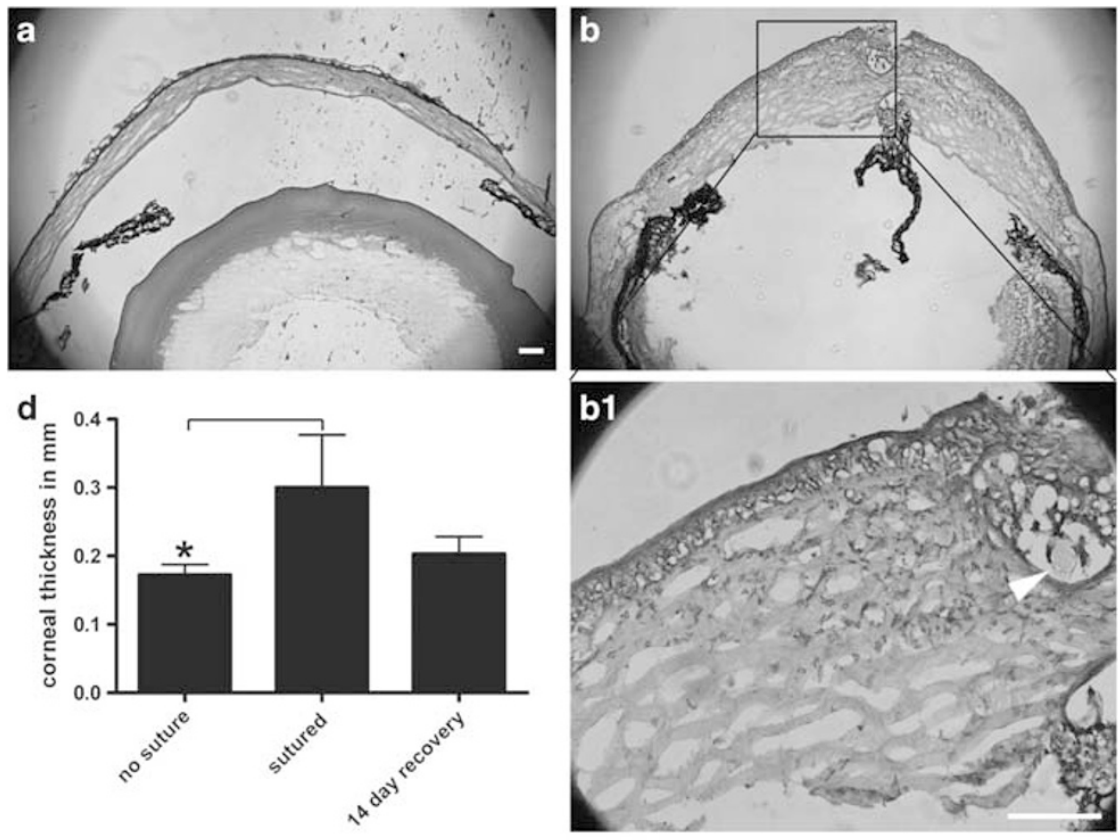
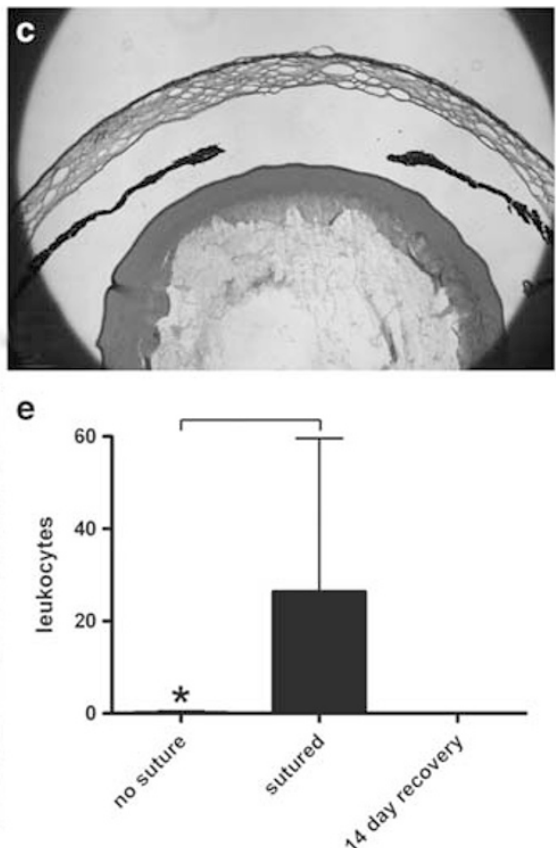

Figure 1 (a-e) Changes in corneal health and inflammation during suture placement and 14-day recovery. Images show H\&E-stained mouse cornea with no suture (a), sutured (b), and 14-day recovery (c). Higher resolution microscopy showed inflammatory cells and collagen hypertrophy in sutured cornea (b1). Suture was identified with white arrowhead (b1). Suture placement induced corneal thickening and collagen hypertrophy (d) and inflammatory cell infiltrate (e) that resolved after suture removal and 14-day recovery. Images (a-c) were obtained at $\times 100$ and (b1) was obtained at $\times 400$. Size bar is $100 \mu$ m. The images were representative of four independent experiments with similar results. Histogram data are mean value \pm s.d and includes six to eight mice in each group. Brackets between groups indicate statistical significance. The asterisk indicates the control group. 

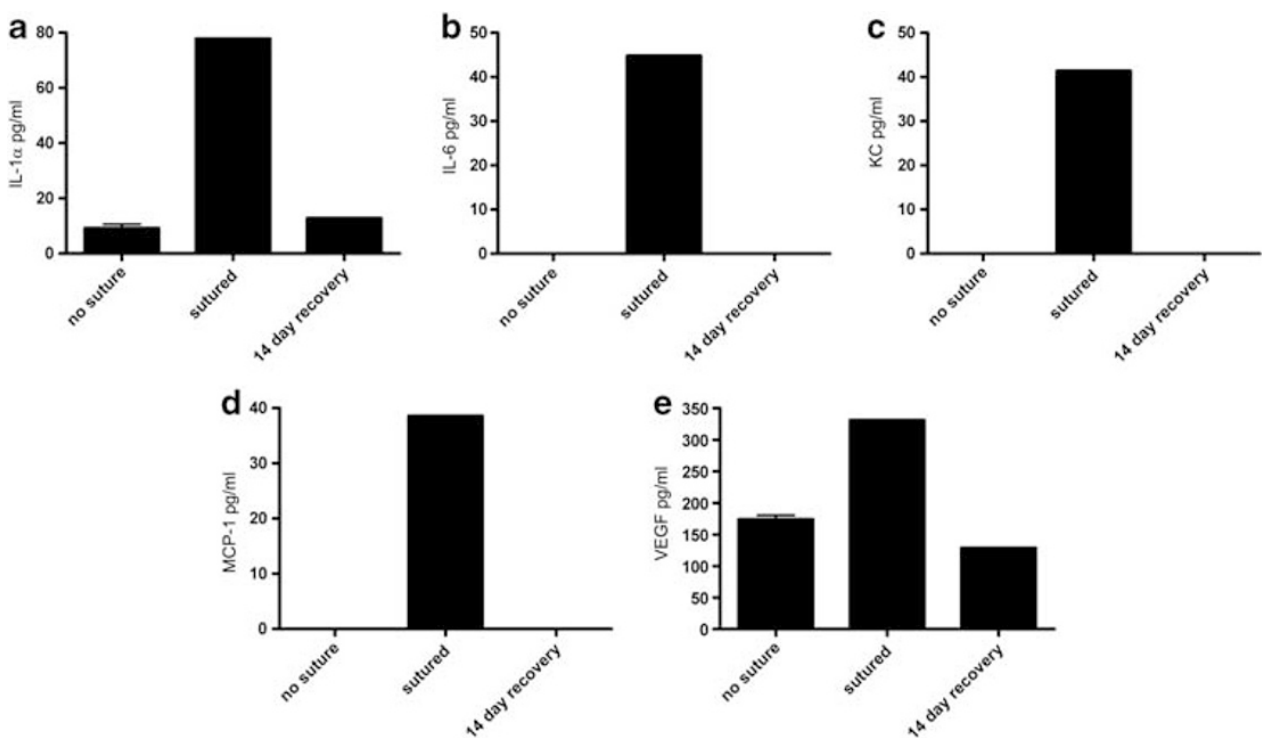

Figure 2 (a-e) Increased inflammatory cytokine expression (IL-1 $\alpha$, IL-6, KC, MCP-1, and VEGF) was quantified in sutured corneal that resolved after suture removal and 14-day recovery. Histogram data are mean value \pm s.d. Error bars are shown but in some cases are small and not seen. The results of one of the two independent experiments with similar results are shown.
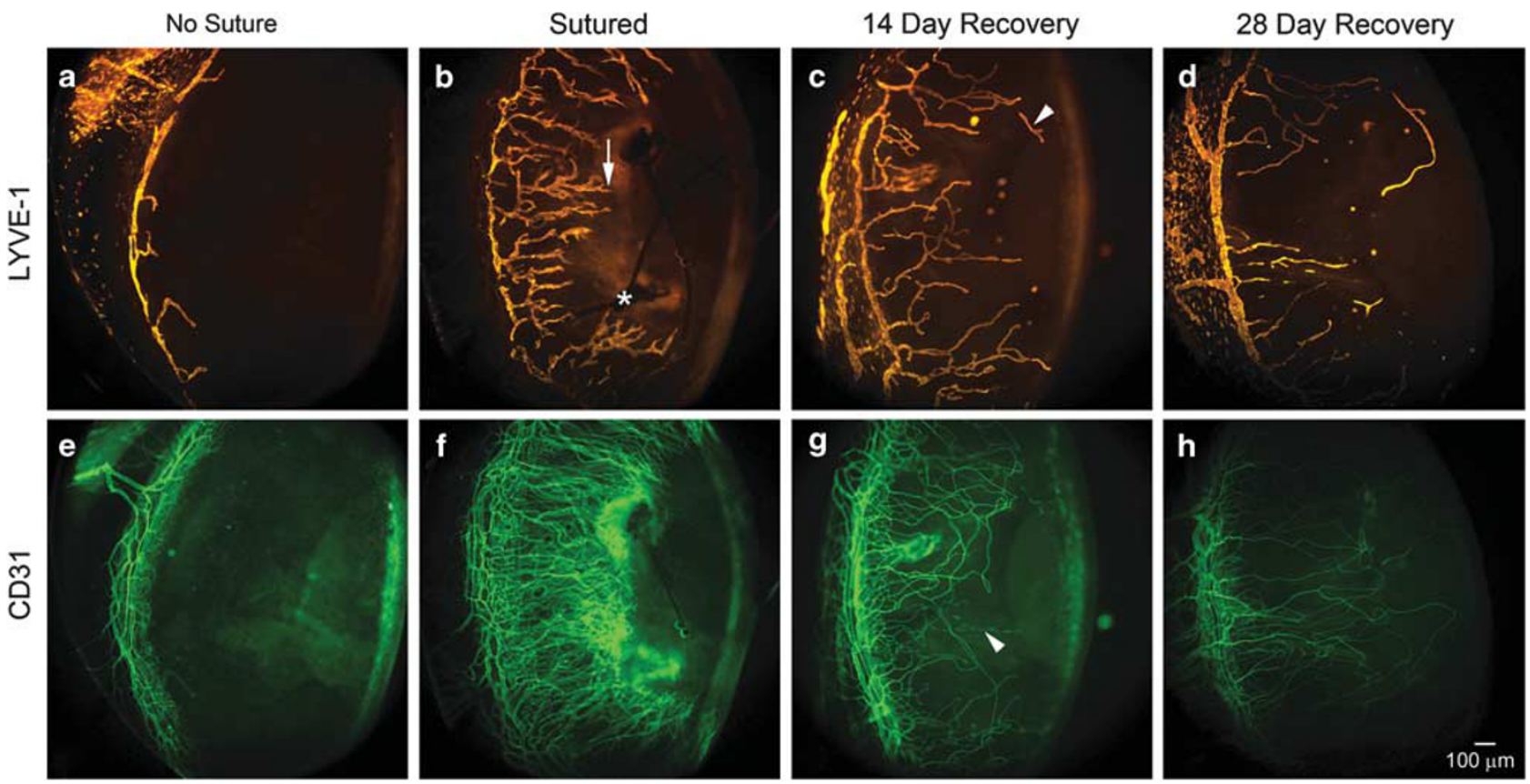

Figure 3 (a-h) Distinct morphologic features in regressed lymphatic vessels. Images were one-half corneal whole mounts with limbal vessels to the left and the central cornea to the right. Limbal lymphatic and blood vessels were visualized adjacent to the cornea (a, e). Lymphatic and blood vessel growth was visualized 7 days after suture placement (b, f). Sprouting lymphatic vessels (arrow) and the suture (asterisk) were identified (b). After suture removal and a 14- or 28-day recovery, morphologic changes were detected in blood and lymphatic vessels. Regressed lymphatic vessels showed loss of lymphatic sprouts, bulbous termini, vessel thinning, and fragmentation (arrowheads in $\mathbf{c}$ and $\mathbf{g}$ ). All images are $\times 100$. The size bar is $100 \mu \mathrm{m}$. The results are representative of more than four independent experiments.

blood vessel growth (Figures $3 b$ and $f$ ). We identified one lymphatic sprouts that appeared to be migrating toward the sutures positioned centrally (Figure $3 b$ ). Blood vessel growth was dense (Figure 3f). Suture removal followed by 14- or 28day recovery induced a phenotype of lymphatic vessel thin- ning, loss of sprouts, bulbous vessel termini, and vessel fragmentation (Figures $3 \mathrm{c}, \mathrm{d}, \mathrm{g}$, and $\mathrm{h}$ ). We termed this phenotype lymphatic vessel regression.

To quantify morphologic vessel changes, we overlaid a rectangular grid measuring $200 \times 400 \mu \mathrm{m}^{2}$ on each corneal 
image adjacent to the constitutively expressed limbal lymphatic vessels. Each square within the grid was $40 \mu \mathrm{m}$ on a side. For lymphatic density measurements, we counted the number of grid squares occupied by a lymphatic vessel wall, lumen, or sprout. We expressed the data as mean relative area of the total grid occupied by lymphatic vessels \pm s.d. We quantified a significant increased in lymphatic vessel density after suture placement that did not change significantly during corneal recovery (Figure 4a). We quantified the number of lymphatic vessel sprouts, defined as a terminal conical projection with a length of least $10 \mu \mathrm{m}$ within the grid. We expressed the data as a mean number of sprouts/ grid \pm s.d. A significant increase in lymphatic vessel sprouts was detected after suture placement that decreased $>90 \%$ during corneal recovery (Figure $4 \mathrm{~b}$ ). During corneal recovery, the number of lymphatic vessel fragments increased over time significantly (Figure 4c).

The morphology of regressed lymphatic vessels suggested that these vessels had distinct functional properties potentially related to corneal inflammation and mechanisms of repair. We designed studies to examine phenotypic and functional differences between regressed and newly synthesized lymphatic vessels.

\section{Phenotypic and Functional Properties of Regressed Lymphatic Vessels}

Using IF microscopy and high-resolution confocal microscopy, we detected differences in LYVE-1, CD31, and VE-cadherin expression in regressed compared with newly synthesized lymphatic vessels. Constitutively expressed limbal lymphatic vessels had little or no detectable CD31 or VE-cadherin expression (Figures 5a and f). Sprouting lymphatic vessels in sutured cornea express LYVE-1 and CD31 (Figures $5 \mathrm{~b}$ and $\mathrm{d}$ ), or VE-cadherin uniformly (Figures $5 \mathrm{~g}$ and i). In contrast, lymphatic vessels undergoing regression had discontinuous button-like expression of CD31 (Figures $5 \mathrm{c}$ and e) or VE-cadherin (Figures $5 \mathrm{~h}$ and $\mathrm{j}$ ). There were significant quantitative differences in CD31 or
VE-cadherin buttons/per unit of regressed lymphatic vessel (Figures $5 \mathrm{l}$ and $\mathrm{m}$ ). Regions of regressed lymphatic vessels had decreased LYVE-1 and CD31 or VE-cadherin expression (Figures $5 \mathrm{c}$ and $\mathrm{h}$, arrowheads). There was a significant number of LYVE-1 discontinuities $/ \mathrm{mm}$ of regressed lymphatic vessels (Figure 5k).

Leukocyte trafficking via lymphatic vessels is thought to contribute to immunity by facilitating antigen and cellular transport to regional lymph nodes. Migrating leukocytes have been shown to pass through cell/cell junctions in lymphatic capillaries or enter higher order collecting lymphatic vessels through dynamic pores. ${ }^{10,11}$ We visualized MHC-II-positive cells docking at the lymphatic sprout and within the lumen of newly synthesized lymphatic cells (Figures 6a-c). Pseudopods from MHC-II-positive cells interfaced with lymphatic vessels and lymphatic vessel filopodia were visualized extending toward MHC-II-positive cells (Figures 6a-c; Supplementary Figure 1). In a given cornea, there was a variable number of MHC-II-positive cell/lymphatic vessel interactions (Figure 7b). Although we detected MHC-II-positive cells in 14-day recovery cornea, we did not visualize or quantify MHC-IIpositive cells docking, trafficking within, or otherwise interfacing with regressed lymphatic vessels (Figures $6 d$ and $7 a-c$ ).

\section{Higher Levels of VEGF-A and -C mRNA in Recovered Corneal Tissue}

Increased VEGF-A, VEGF-C, and TGF- $\beta$ mRNA was quantified in recovered corneal tissue. MMP-10 mRNA increased significantly after suture placement and returned to premorbid levels during corneal recovery. TNF- $\alpha$ mRNA levels did not change significantly (Figure 8).

\section{DISCUSSION}

The findings presented in this manuscript demonstrate that newly synthesized lymphatic vessels stimulated by inflammation undergo an orderly program of lymphatic vessel regression during corneal recovery. The measurements of corneal health documented corneal recovery. These studies
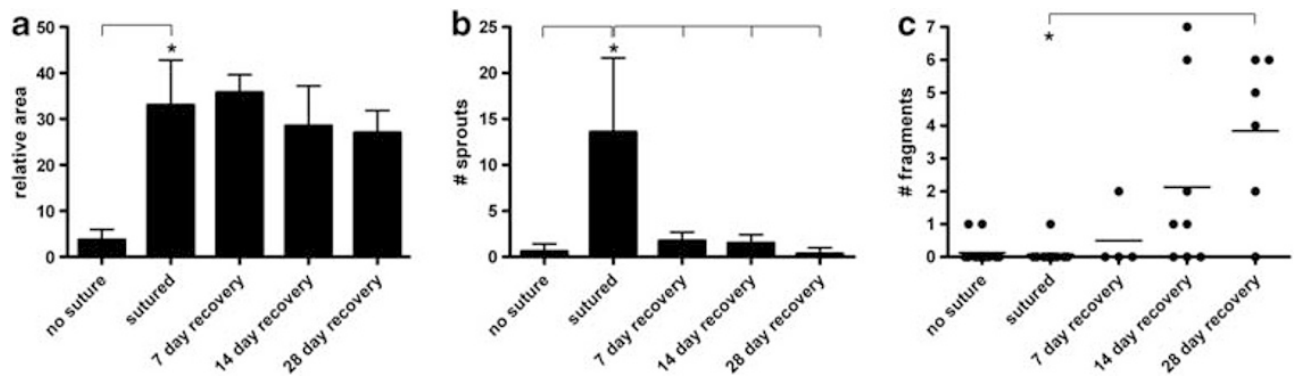

Figure 4 (a-c) Cornea recovery was associated with changes in lymphatic vessel sprouting and fragmentation, but not density. Using morphologic analysis, we demonstrated a significant increase in corneal lymphatic vessel density after suture placement that persisted after suture removal and corneal recovery for 14 or 28 days (a). The number of lymphatic vessel sprouts increased after suture placement and decreased sharply after suture removal and corneal recovery $(\mathbf{b})$. The mean relative lymphatic vessel area or number of sprouts is shown \pm s.d. Using similar techniques, we detected corneal lymphatic vessel fragmentation after suture removal and corneal recovery (c). These figures were composite data from four independent experiments with four to six mice per group with one exception; we used four mice in the 7-day recovery experiments. Brackets between groups indicated a statistically significant difference. The asterisk indicates the control group. 

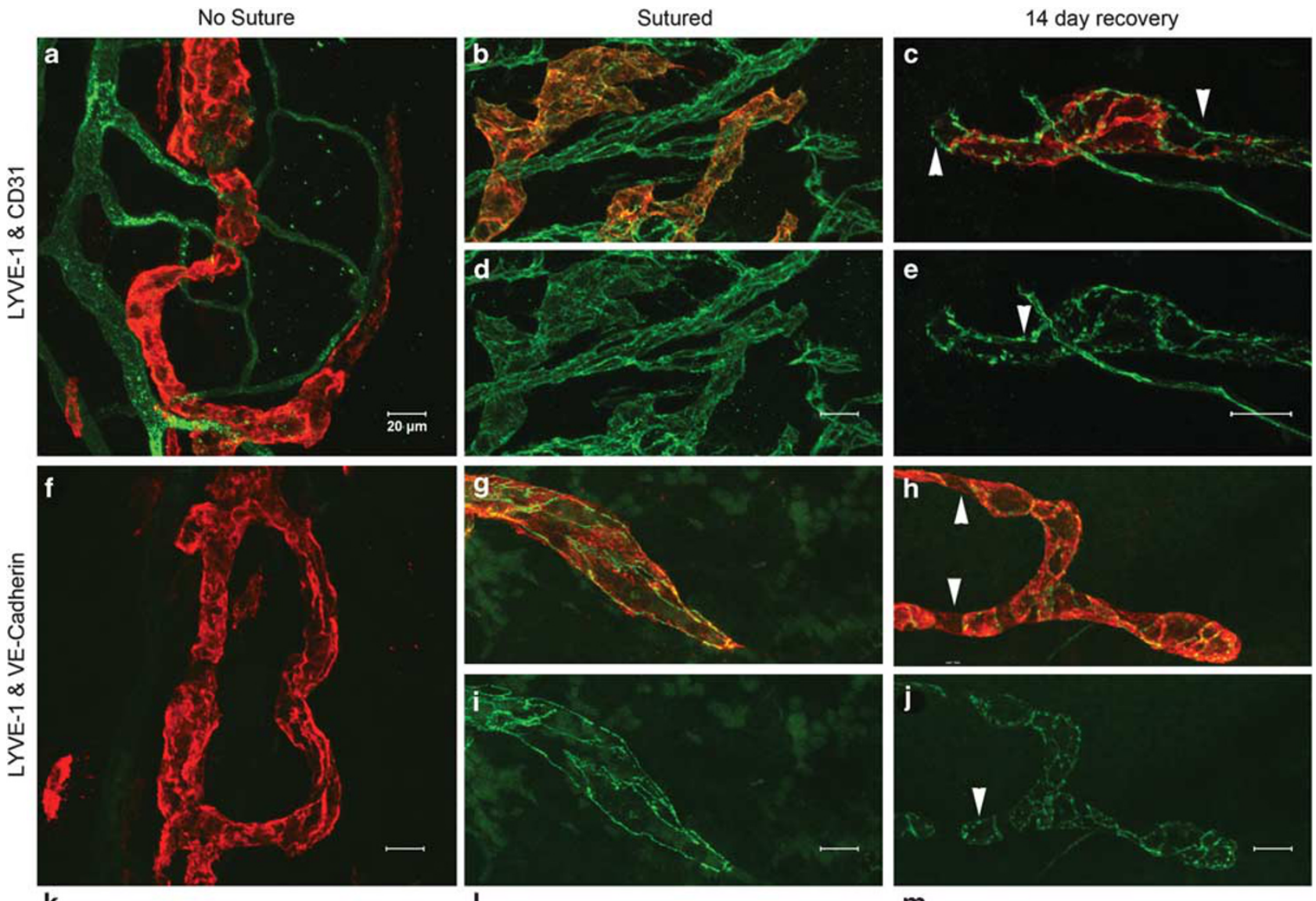

k

I

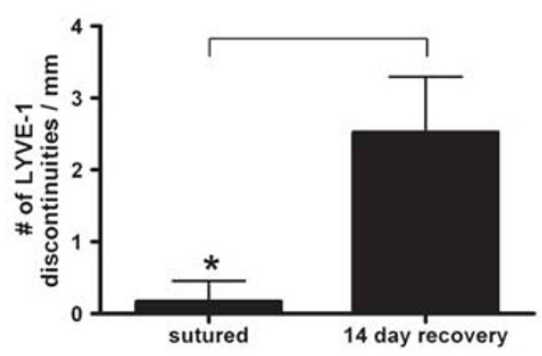

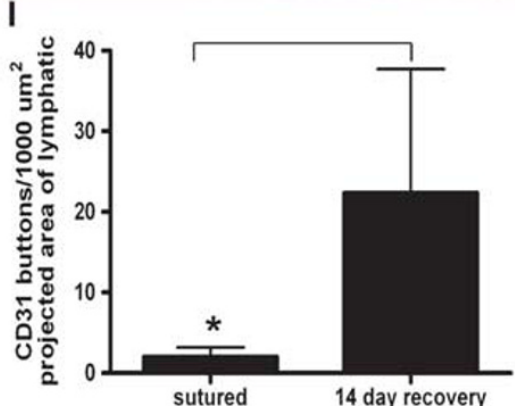

m

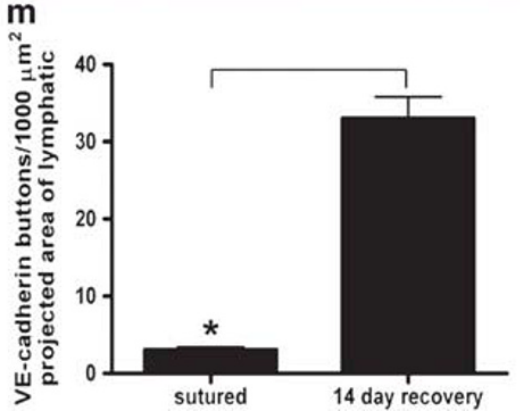

Figure 5 (a-m) Discontinuous button CD31 and VE-cadherin expression in regressed lymphatic vessels. Little CD31 or VE-cadherin expression (green) was detected in limbal lymphatic vessels (red) in unsutured cornea (a or f). Nearly continuous CD31 or VE-cadherin expression (green) was detected in newly synthesized lymphatic vessels (red) (b, $\mathbf{d}$ and $\mathbf{g}$, i). The images in $(\mathbf{b}, \mathbf{d})$ were identical fields. In (b), both red and green channels are shown to identify the lymphatic vessels. In (d), only the green channel (CD31) is shown to demonstrate the continuous expression of CD31. Similar data presentation in (g, i) shows continuous expression of VE-cadherin in newly synthesized lymphatic vessels. Discontinuous button-like CD31 or VE-cadherin expression (green) was detected in lymphatic vessels undergoing regression. The images in (c, e) were identical fields. In (c), both red and green channels were shown to identify the lymphatic vessels. In (e), only the green channel (CD31) was shown to demonstrate the discontinuous expression of CD31 (arrowheads in (c, e)) in regressed lymphatic vessels. Similar data presentation in $\mathbf{( h}, \mathbf{j})$ shows discontinuous expression of VE-cadherin in regressed lymphatic vessels (arrowhead in (j)). A statistically significant difference in CD31 or VE-cadherin buttons was quantified in regressed lymphatic vessels $(\mathbf{I}, \mathbf{m})$. There were numerous regressed lymphatic vessel regions that had decreased LYVE-1 and/or CD31 or VE-cadherin expression (k, examples are shown with arrowheads in (c, $\mathbf{h}$ )).

The histogram data were mean value \pm s.d and was representative of three independent experiments with three to four mice in each group. The asterisk indicates the control group.

allowed the interpretation of lymphatic vessel regression in the context of corneal recovery. Other investigators have documented lymphatic vessel regression during corneal development, ${ }^{12}$ after corneal inflammation, ${ }^{8}$ and as a risk factor for corneal transplant rejection in a preclinical model. ${ }^{7}$ This work compliments those findings and is the first report to demonstrate morphologic, phenotypic, and functional differences in regressed lymphatic vessels. The concept of lymphatic vessel regression is a topic of debate in the literature. New tracheal or corneal lymphatic vessel growth stimulated by pathogens has been shown to persist after the resolution of the infection. ${ }^{2,5}$ The persistent nature of 

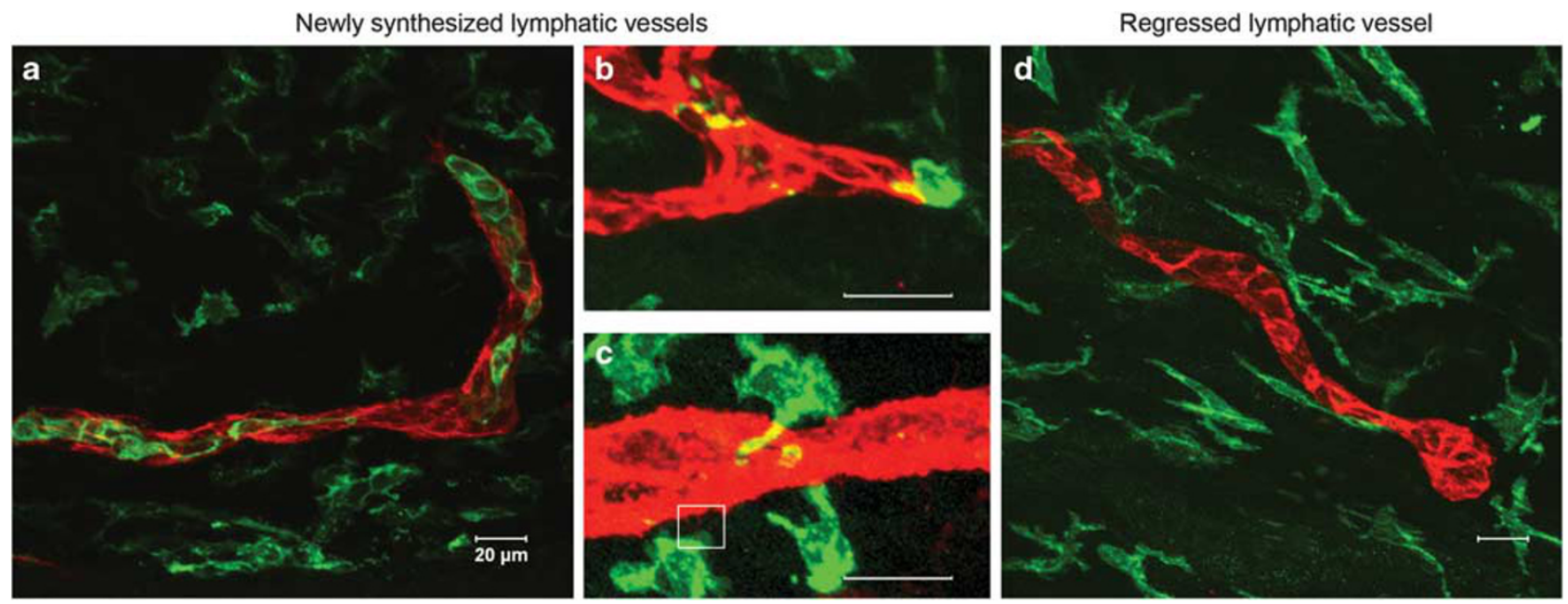

Figure 6 (a-d) Regressed lymphatic vessels excluded MHC-II-positive cells. MHC-II-positive cells dock and traffic within newly synthesized lymphatic vessels. Polarized MHC-II-positive cells were visualized within the lumen of newly synthesized lymphatic vessels (a), interfacing at the sprout tip (b), and extending pseudopods toward the lymphatic stalk (c). Fine lymphatic vessel filopodia extending to MHC-II-positive cells were identified in some cases (shown in (c), boxed region). MHC-II-positive cells were not visualized interfacing or within regressed lymphatic vessels. In all images, size bar is $20 \mu \mathrm{m}$. These representative images were obtained from more than six mice in each group.
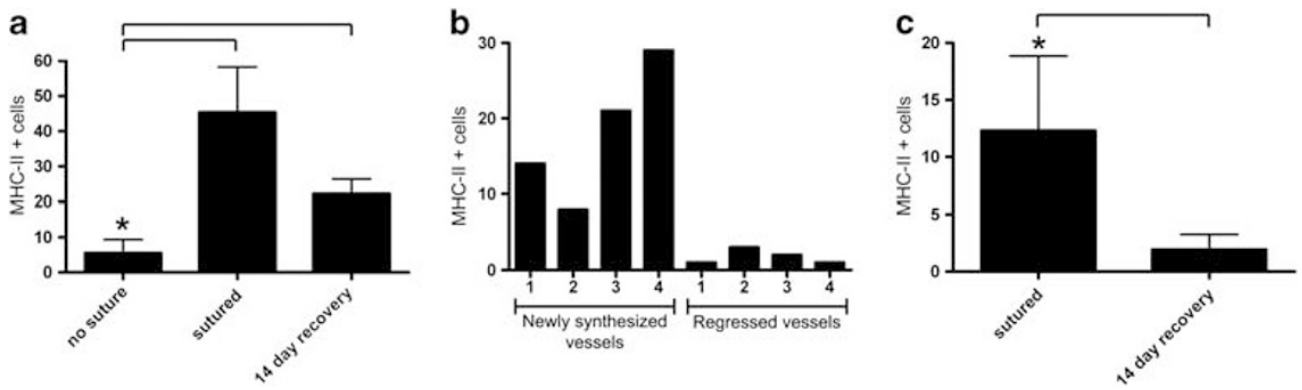

Figure $7(\mathbf{a}-\mathbf{c})$ There is a greater and variable number of MHC-II-positive cells interfacing or within newly synthesized compared with regressed lymphatic vessels. Corneas from unsutured, sutured, and 14-day recovery mice have MHC-II-positive cells (a). In an individual, the number of MHC-II-positive cells docked or within each newly synthesized lymphatic vessel was variable (range 7-28 (number of MHC-Il positive cells)). There were only a few MHC-II-positive cells in contact and none within regressed lymphatic vessels (b). Within a population, there was a significant increase in the number of MHC-II-positive cells interfaced or within newly synthesized lymphatic vessels compared with regressed lymphatic vessels. (a) The histogram data are mean number of MHC-II-positive/ $\times 400$ field \pm s.d. (b) The number of MHC-II-positive cells per individual vessel was quantified to show vessel-to-vessel variability. (c) The histogram data show the mean number of MHC-II-positive cells \pm s.d. docked or within newly synthesized or regressed lymphatic vessels from 16 mice by analyzing at least two corneal fields from each mouse. Brackets indicate a statistically significant difference between groups. These results are representative of three independent experiments. The asterisk indicates the control group.
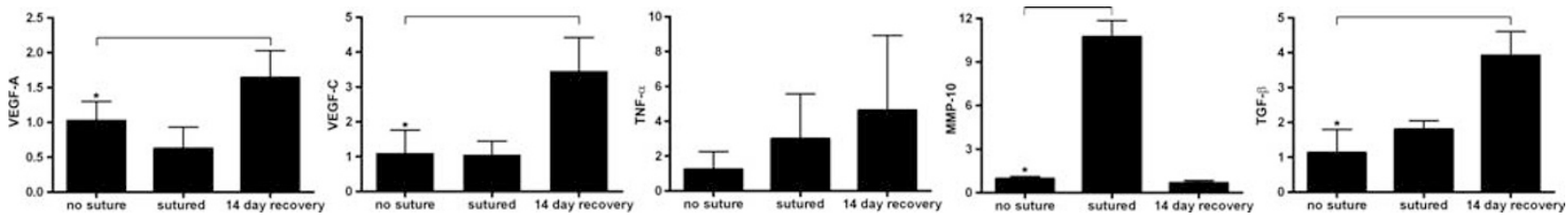

Figure 8 Identification of candidate factors regulating lymphatic vessel growth or regression. VEGF-A, VEGF-C, and TGF- $\beta$ mRNA levels were increased significantly during corneal recovery. MMP-10 mRNA increased during suture placement and decreased during corneal recovery. Levels of TNF- $\alpha$ mRNA did not change significantly. Data were normalized using GAPDH. The histogram bars are mean fold mRNA level normalized to GAPDH \pm s.d. The data were pooled from two groups of mice with three mice per group. The asterisk indicates the control group.

pathogen-induced inflammation makes it difficult to demonstrate whether lymphatic vessels persist without regression or are driven continually to expand by chronic inflammation. Lymphatic vessel regression over many months has been documented in the cornea after inflammation. ${ }^{8}$ In this study, we showed similar findings in that the relative density of the lymphatic vessels did not change significantly during corneal recovery. However, there were numerous identifiable differences in regressed lymphatic vessels, which suggested that these vessels function differently 
than newly synthesized lymphatic vessels. Notably, the loss of sprouts, the appearance of bulbous termini, vessel fragmentation, button-like expression of CD31 and VE-cadherin, and the exclusion of MHC-II leukocytes indicate a unique function for regressed lymphatic vessels.

It could be argued that corneal lymphatic vessel regression is a specialized property to minimize corneal inflammatory reactions (immune privilege) to facilitate transparency and visual acuity. We identified lymphatic vessel fragments as a portion of the vessels comprising the relative density. Lymphatic vessel fragmentation is process that has been identified by others, ${ }^{6}$ and does not seem to be a mechanism used by the blood vascular system. ${ }^{13}$ We suspect that vessel fragmentation has some relationship to the regressed lymphatic vessel regions with decreased LYVE-1 expression and the button-like expression pattern of CD31, and VE-cadherin expression, a phenotype that was recognized by Baluk et al. ${ }^{9}$ Alterations in the expression of CD31 or VE-cadherin on lymphatic endothelial cells are thought to have a role in cellular and fluid transport function in distal lymphatic vessels. The molecular mechanisms underlying lymphatic vessel fragmentation are not understood. Apoptosis via cues from the stroma or other cytokines are candidate possibilities.

These findings raise the question of whether lymphatic vessel regression is an active process or a passive process that develops in the absence of factors driving lymphangiogenesis. ${ }^{14}$ Unexpectedly, higher mRNA levels of the prolymphangiogenic factors, VEGF-A and VEGF-C, and the anti-lymphangiogenic factor TGF- $\beta^{15}$ were detected in recovered cornea. Expression of MMP-10, a recently identified protease expressed uniquely by lymphatic endothelial cells, ${ }^{16}$ was detected in suture cornea. The finding of both pro- and anti-lymphangiogenic factors in recovered cornea suggests an active potentially complex mechanism that regulates lymphatic vessel regression during the resolution of corneal inflammation. These factors may have a critical role in the reactivation of the lymphatic vasculature during subsequent inflammatory episodes.

\section{Regressed Lymphatic Vessels Excluded MHC Class II-Positive Leukocytes}

It is well known that leukocytes enter lymphatic vessels and traffic through higher order lymphatic collecting vessels en route to draining regional lymph nodes. This series of events has been described as the afferent arc of immunity and is thought to be critical in the generation of an effective immune response. ${ }^{17}$ Despite the acknowledged significance of this process, there are major limitations in our understanding of the mechanistic details of leukocyte transport within distal lymphatic vessels. Here, we show that regressed lymphatic vessels do not transport MHC-II-positive leukocytes; a significant functional distinction between regressed and newly synthesized lymphatic vessels. MHC-II-positive leukocytes were visualized docking at the sprout tip or within the lumen of some but not all newly synthesized lymphatic vessels. This is conceptually similar to the work of others. $^{9,18,19}$ A different consideration is that possibility that MHC-II-positive macrophages were physically integrating/ transdifferentiating into newly synthesized lymphatic vessels as others have described. ${ }^{20}$ We visualized MHC-II-positive cells with pseudopod extensions toward the stalk region of newly synthesized lymphatic vessels. This is reminiscent of the recent work by Pflicke and Sixt, ${ }^{11}$ describing dynamic portals on lymphatic vessels designed to accommodate leukocyte intravasation. Within the lymphatic lumen, we visualized a polarized 'amoeboid' morphology of some of the MHC-II-positive cells. A cytoplasmic extension was directed toward the lymphatic tip and the nucleus was localized opposite to this extension. Based on this morphology it could be argued that the MHC-II-positive cells were migrating toward the lymphatic tip ${ }^{21}$ rather than proximally as conventional wisdom would suggest. Based on these observations, we are willing to consider that the lymphatic vasculature may be more than strictly a unidirectional system.

\section{Clinical Implications}

Although the precise mechanisms are unclear, many lines of evidence demonstrate that lymphangiogenesis has a pathologic effect on the course of corneal and non-corneal inflammatory disease conditions. Failure or perturbation of lymphatic vessel regression may facilitate the persistence of newly synthesized lymphatic vessels, which may promote ongoing inflammatory responses and inadequate tissue repair mechanisms. We hypothesize that lymphatic vessel regression is an important and perhaps an essential regulator of tissue homeostasis and repair. Understanding the relationship between the lymphatic vasculature and the inflammatory response represents a major achievement that will likely improve the management of clinical inflammatory diseases.

Supplementary Information accompanies the paper on the Laboratory Investigation website (http://www.laboratoryinvestigation.org)

\section{ACKNOWLEDGEMENTS}

This work was supported by the Nebraska Tobacco Settlement Fund. We acknowledge the use of the confocal microscope at the Creighton University Integrative Biomedical Imaging Facility. This facility, supported by the CU Medical School and the National Center for Research Resources (NCRR, P20 RR16469), was constructed with support from the NCRR (C06 RR17417-01), a component of the National Institutes of Health (NIH). This investigation is solely the responsibility of the authors and does not necessarily represent the official views of $\mathrm{NCRR}$ or $\mathrm{NIH}$.

\section{DISCLOSURE/CONFLICT OF INTEREST}

The authors declare no conflict of interest.

1. Dietrich $T$, Bock F, Yuen D, et al. Cutting edge: lymphatic vessels, not blood vessels, primarily mediate immune rejections after transplantation. J Immunol 2010;184:535-539.

2. Wuest TR, Carr DJ. VEGF-A expression by HSV-1-infected cells drives corneal lymphangiogenesis. J Exp Med 2010;207:101-115. 
3. El-Chemaly S, Malide $D$, Zudaire $E$, et al. Abnormal lymphangiogenesis in idiopathic pulmonary fibrosis with insights into cellular and molecular mechanisms. Proc Natl Acad Sci USA 2009:106:3958-3963.

4. Sakamoto I, Ito $Y$, Mizuno $M$, et al. Lymphatic vessels develop during tubulointerstitial fibrosis. Kidney Int 2009;75:828-838.

5. Baluk $P$, Tammela $T$, Ator $E$, et al. Pathogenesis of persistent lymphatic vessel hyperplasia in chronic airway inflammation. J Clin Invest 2005;115:247-257.

6. Yao LC, Baluk P, Feng J, et al. Steroid-resistant lymphatic remodeling in chronically inflamed mouse airways. Am J Pathol 2010;176:1525-1541.

7. Bachmann BO, Luetjen-Drecoll E, Bock F, et al. Transient postoperative vascular endothelial growth factor (VEGF)-neutralisation improves graft survival in corneas with partly regressed inflammatory neovascularisation 27. Br J Ophthalmol 2009;93:1075-1080.

8. Cursiefen C, Maruyama K, Jackson DG, et al. Time course of angiogenesis and lymphangiogenesis after brief corneal inflammation. Cornea 2006;25:443-447.

9. Baluk $\mathrm{P}$, Fuxe J, Hashizume $\mathrm{H}$, et al. Functionally specialized junctions between endothelial cells of lymphatic vessels. J Exp Med 2007;204: 2349-2362.

10. Johnson LA, Clasper S, Holt AP, et al. An inflammation-induced mechanism for leukocyte transmigration across lymphatic vesse endothelium. J Exp Med 2006;203:2763-2777.

11. Pflicke $\mathrm{H}$, Sixt $\mathrm{M}$. Preformed portals facilitate dendritic cell entry into afferent lymphatic vessels. J Exp Med 2009:206:2925-2935.
12. Zhang $\mathrm{H}, \mathrm{Hu} \mathrm{X}$, Tse J, et al. Spontaneous lymphatic vessel formation and regression in the murine cornea. Invest Ophthalmol Vis Sci 2011;52:334-338.

13. Im E, Kazlauskas A. New insights regarding vessel regression. Cell Cycle 2006:5:2057-2059.

14. Tammela T, Alitalo K. Lymphangiogenesis: molecular mechanisms and future promise. Cell 2010;140:460-476.

15. Oka M, Iwata C, Suzuki Hl, et al. Inhibition of endogenous TGF-beta signaling enhances lymphangiogenesis. Blood 2008;111:4571-4579.

16. Steele $\mathrm{MM}$, Schieler $\mathrm{AM}$, Kelley $\mathrm{PM}$, et al. beta1 integrin regulates MMP-10 dependant tubulogenesis in human lymphatic endothelial cells. Matrix Biol 2011;30:218-224.

17. Alvarez D, Vollmann EH, von Andrian UH. Mechanisms and consequences of dendritic cell migration. Immunity 2008;29:325-342.

18. Tripp CH, Haid B, Flacher V, et al. The lymph vessel network in mouse skin visualised with antibodies against the hyaluronan receptor LYVE1. Immunobiology 2008;213:715-728.

19. Randolph GJ, Angeli V, Swartz MA. Dendritic-cell trafficking to lymph nodes through lymphatic vessels. Nat Rev Immunol 2005;5: 617-628.

20. Maruyama II KM, Cursiefen C, Jackson DG, et al. Inflammation-induced lymphangiogenesis in the cornea arises from CD11b-positive macrophages. J Clin Invest 2005;115:2363-2372.

21. Lammermann T, Sixt M. Mechanical modes of 'amoeboid' cell migration. Curr Opin Cell Biol 2009;21:636-644. 